\title{
Wireless Sensor Network for Wildlife Tracking and Behavior Classification of Animals in Doñana
}

\author{
J. P. Dominguez-Morales, A. Rios-Navarro, M. Dominguez-Morales, R. Tapiador-Morales, D. Gutierrez-Galan, \\ D. Cascado-Caballero, A. Jimenez-Fernandez, and A. Linares-Barranco
}

\begin{abstract}
The study and monitoring of wildlife has always been a subject of great interest. Studying the behavior of wild animals is a difficult task due to the difficulties of tracking and classifying their actions. Nowadays, technology allows designing low-cost systems that make these tasks easier to carry out, and some of these systems produce good results; however, none of them obtains a high-accuracy classification because of the lack of information. Doñana National Park is a very rich environment with various endangered animal species. Thereby, this park requires a more accurate and efficient system of monitoring to act quickly against animal behaviors that may endanger certain species. In this letter, we propose a hierarchical, wireless sensor network installed in this park, to collect information about animals' behaviors using intelligent devices placed on them which contain a neural network implementation to classify their behavior based on sensory information. Once a behavior is detected, the network redirects this information to an external database for further treatment. This solution reduces power consumption and facilitates animals' behavior monitoring for biologists.
\end{abstract}

Index Terms-Sensor network, neural network, pattern recognition, 802.15.4, distributed system.

\section{I.INTRODUCTION}

$\mathbf{M}$ ONITORING the behavior of wild animals is a hard technological task to implement. There are some

commercial devices that can track animals using GPS and obtain some vital signs through sensors. The information given consists only of raw data without processing, thus, these solutions are not able to recognize animal patterns with the data obtained from its attached sensors.

This kind of classification is usually done using neural networks, which can detect a specific behavior, such as sleeping, running, copulating, etc, in either a supervised or an unsupervised way, depending on the network requirements. Generally, the computational costs of a neural network are high enough to need specific platforms able to parallelize this information and classify it. Hardware like the board shown in [2] allows us to develop spiking neural networks easily; however, the size and power consumption of this board are extremely high for a sensor network.

MINERVA is a research project whose main target is to study and classify wildlife behavior inside Doñana

The authors are with the Robotic and Technology of Computers Lab., E.T.S. Ingenieria Informatica, University of Seville, 41012 Sevilla, Spain (e-mail: jpdominguez@atc.us.es).
National Park [3]. To achieve this goal, a hierarchical wireless sensor network (WSN) capable of transmitting and storing this information has to be developed and tested inside this park. Moreover, to increment the value of the final product, an embedded system with energy harvesting techniques that will be able to digest the inertial sensor information [4] combined with other sensors (temperature, heart rate, GPS) has been developed in order to classify animal behavior in real time. The project has the additional aim of developing an infrastructure for collecting this information and make it accessible through the Internet. The pattern recognition of the collected data is done in real time using a low-cost implementation of a neural network inside the microcontroller that classifies among five behaviors (feeding, sleeping, walking, jogging and running).

In this manuscript, we present a wireless multisensory network, distributed in collars placed on some animals, which reads data from the sensors and sends them to a mote network, which acts like a router and retransmits these packets to a base station. This base station receives the information through the network and uploads it to a remote server with a database. Researchers can access this data using a web-based user interface and track the animal activity at any time without the necessity of being in Doñana National Park [3], [4]. Moreover, neural network implementation (inside the collar) for behavior classification is compared to classic raw data sending methods in term of power consumption and battery life.

The letter is structured as follows: section II presents the network architecture, every element within and the type of data sent through it. Second, section III presents the testing scenario for the signal coverage and its results. Then, section IV describes the neural network, its efficiency, power consumption and compares it to external classification methods. Finally, section $\mathrm{V}$ presents the conclusions of this work.

\section{Network Topology}

The aim of this network is to gather behavioral information from wildlife with the lowest power consumption possible and store it in a remote server, so researchers can access this data through a website for future studies. This information will be collected and sent by the collars, while a mote network will transmit it to the corresponding base station located in the park, as can be seen in Fig. 1.

The network architecture used is $\mathrm{ZigBee}(2.4 \mathrm{GHz})$, which is an open global standard of the IEEE 802.15.4 MAC/PHY [5].

In our network topology we define 3 different device types: base station (coordinator), motes (routers) and collars (end devices), respectively. The sniffer is not a working device in the network; it is used to find lost devices that could have gone 


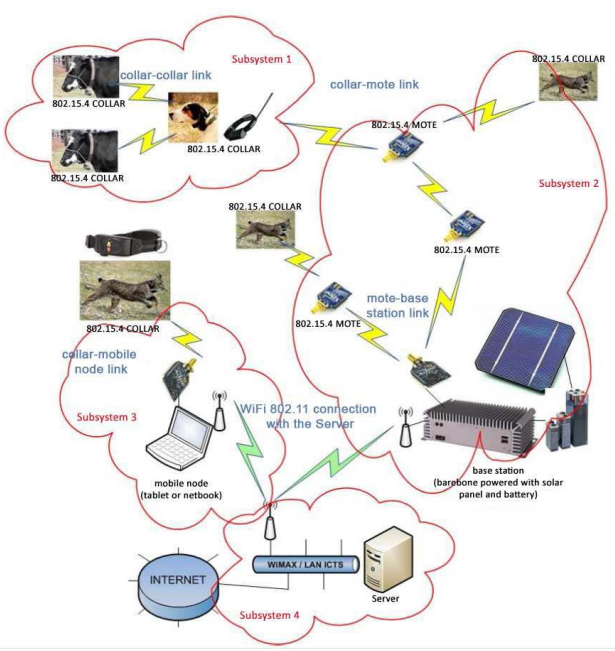

Fig. 1. Network topology architecture.

outside the network coverage area. Routers must join the base station before transmitting and receiving data. This type of devices allows others to join the network. Finally, the collars must join the ZigBee Personal-Area-Network (PAN) too; however, this does not allow other devices to join the network. The four elements implemented are presented next.

\section{A. Collars}

They are placed on the target animals and their role is to collect information from the environment using multiple sensors. Those sensors are a GPS (location and time) and an accelerometer. This data will allow recognizing behavioral patterns. The periodical measurements of each sensor are done using a low power consumption microcontroller [6].

The process to obtain data from each device is organized as a task. This makes it possible to set the microcontroller to sleep mode when there are no devices receiving data (this increases battery life and avoids using interruption signals that could block the microcontroller). A special task monitors the power from the collar, disabling devices to increase battery life.

The radio module is used to transmit data through the network to the nearest points inside its action radius (standard $\mathrm{ZigBee} 2.4 \mathrm{GHz}$ at $250 \mathrm{kbps}$ ). If the signal does not reach a valid point to transmit data, i.e. the animal is outside the network coverage area, the device carries an SD card where the information is stored (it can be accessed later), avoiding data loss.

The collar does not spend the whole time transmitting information in a continuous way, but it receives variation alerts from the different sensors; these variations indicate the information the device has to send, although a periodicity limit has been established to reduce transmit power consumption. To improve its battery life, a neural network has been implemented inside each collar. This pre-trained network processes data from sensors, obtains the animal behavior and sends it to the network. The periodicity of these transmissions and the data acquisition are evaluated on section IV in order to measure the benefits obtained using this solution.

\section{B. Motes}

These are router devices which are placed around the base station to cover the area where the animal usually moves. The device is powered by a set of batteries and a solar cell in order to have unlimited duration of power supply, with the idea of placing the device on any tree, rock, etc. The mote receives the information from the collar joined to it and routes it to the base station.

\section{Base Stations}

There are some special devices inside every sensor network that are responsible of providing consistence to the network itself: base stations in our case. Their tasks include, among others, to serve as a WSN network coordinator and send packets to the database server. In this implementation, the base station receives data packets from collars inside its action radius and retransmits them to a remote web server via Doñana National Park's Wi-Fi connection. Some additional functionalities have been added, focused on monitoring the park's environmental conditions and informing the central web server about them.

The sensors and devices placed in the BridgeBoard are: a temperature and humidity sensor, an inertial measurement unit to detect if the base station has tipped over, an ambient light sensor, a current sensor IC to monitor battery status and a GPS to position the base station in the park.

The computer runs a software tool in the background that reads data from the sensors and, after obtaining the interesting information (location, time and accelerometer) from the data frames, sends it packetized to the database.

\section{802.15.4 Sniffer Device}

The mobile application allows localizing the collars and base stations in the field, in order to configure the position of new motes, or tracking animals through the proper GPS data from each collar or by RSSI and a directional antenna when the GPS does not function. These requirements force to implement the radio layer of the mobile application as a sniffer for 802.15.4 packets, which captures all the data packets sent by all the nodes of the network and is capable of analyzing and obtaining information about signal strength and location. This strategy assumes that in free space, multipath effects are scarce and faint, making the location of radio nodes easier [9]. This sniffer is connected to a portable device through an USB port, used to send information of every ZigBee packet that it is detected on a particular channel, which can be selected by the user. This way, the RSSI, collar ID and other important information can be consulted in the software application and determine the relative position of the animal from the sniffer. The collars must connect to the base stations in order to send their data to the main server, which will send them to the database. The base stations are connected in a tree-fashion to the rest of the nodes of the network. When the collars are connected to the base station, they send their location, which can be used by the mobile node to locate them. Note that the mobile node can see the orphan node based on the request for connection data packets. These packets can be used to locate them using a directional antenna and a moving average media 


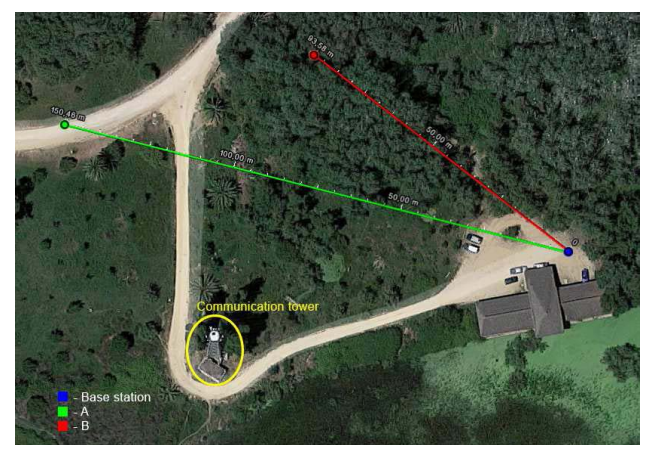

Fig. 2. Satellite view of the area were the test was performed. The blue dot represents the base station. A and B paths are colored in green and red, respectively.

filter [11] based on a weighted combination of RSSI and the number of packets received per time unit.

The mobile node is composed of an antenna, an NMEA GPS integrated with a radio module, and a tablet device. GPS and 802.15.4 frames are collected by the adaptation layer that interprets the 802.15.4 packets and sends information about location, time of reception, RSSI and MAC to the application layer, together with the proper location.

\section{Study of the Signal Coverage on the Network}

The signal coverage on the network is an important issue that was discussed previously. The study developed to quantify the collar coverage in the park was tested with the animal moving in two different paths. The main aim of this scenario was to detect RSSI variation, GPS information and ZigBee coverage. The result will determine the network distribution and the correct performance of the selected elements.

A very important factor in a WSN is the signal coverage of every element within it. Every element needs to be connected properly to the next hop to be able to transmit the information to the final destination. Since the collar needs to be able to send sensor information to base stations in order to upload this to the database, this communication channel must be guaranteed at a certain point. The mote network will be helpful in this task, but additionally the signal coverage for the collar must be known so the number of motes that should be placed in the park for the appropriate transmission can be estimated.

The test consisted of measuring the signal coverage distance between a collar and a base station by tracking the collar GPS information until it lost connection with the base. Two different paths were followed to test this approach: one mostly clear with few vegetation (A) and the other one with high density vegetation between the collar and the station (B). Both paths can be seen in Fig. 2. To measure this, an XBee router was connected to a tablet in the area where the base station was settled. This tablet was running an application that showed the sensor information of the collar, which was held while walking away from the base station. For these tests, the worst case scenario was chosen: the area was not clear and both paths had a communication tower close to the base station and the collar, what could reduce the signal coverage. Next visits to Doñana will be focused on measuring the signal coverage in a more realistic scenario, which would provide more favorable values than the worst case (described next).
In the A path test, the collar lost signal after walking away 150 meters from the router, and right before that moment the RSSI was $-80 \mathrm{~dB}$. In the B path test the collar did not lose signal, even though the vegetation density in that area was very high. The GPS data frames transmission in this last test was slower than in the first one, but still correct, and the RSSI was oscillating between -35 and $-50 \mathrm{~dB}$. The sniffer was also tested using these two scenarios and the results were almost the same in both cases. It also lost signal in the same spot as the collar and it was able to collect sensor information within the dense vegetation area, showing a very good outcome.

During these tests, the base station was running and able to receive data frames from the collar and upload them to the database; thus, the communication was achieved without any kind of problem and the data is accessible through the website.

\section{Neural Network Implementation AND POWER CONSUMPTION ANALYSIS}

The work has presented the network topology installed in Doñana National Park and its viability inside the perimeter using the signal coverage study. However, the novelty of this work lies on the in-collar behavior classification.

Nowadays, tracking and classification systems for wildlife used in Doñana National Park obtain positional information using a GPS and transmit it via GSM (by SMS). However, to reduce the power consumption, the position is obtained between two and five times a day. These solutions are not enough for biologists' interests: they need more information to recognize animal behaviors. To solve this lack of information, two solutions can be implemented: the system can be adapted to transmit information more regularly (since communications consume in average more than $80 \%$ of battery life, this option is inefficient); or, on the other hand, this information can be processed in a local way in order to classify the animal behavior and transmit only the behavior itself. Therefore, instead of sending the information after every sensor read, the communication to the network only occurs after several sensor reads; this fact increases battery life but keeps the information of animal behaviors. The classification method consists of a pre-trained neural network and it is described next.

\section{A. Neural Network}

It consists of a Multi-Layer Perceptron (MLP) neural network (NN) that is most commonly used with the backpropagation algorithm. The topological structure of the MLP consists of a two-layer feed-forward network, with sigmoid transfer function in hidden layer and soft-max transfer function in output layer. The best performance was obtained using 10 neurons in the hidden layer. The output layer has as many neurons as behaviors to be classified, and the input layer has as many neurons as sensor inputs. The NN architecture and the results of this study are described in [12], obtaining an average result of $80 \%$ hit rate. Thus, this mechanism is feasible to implement as the classification method inside the collar.

\section{B. Power Consumption Study}

Each sensor has been evaluated separately as well as the full collar. The system remains in a "deep-sleep" state until the 


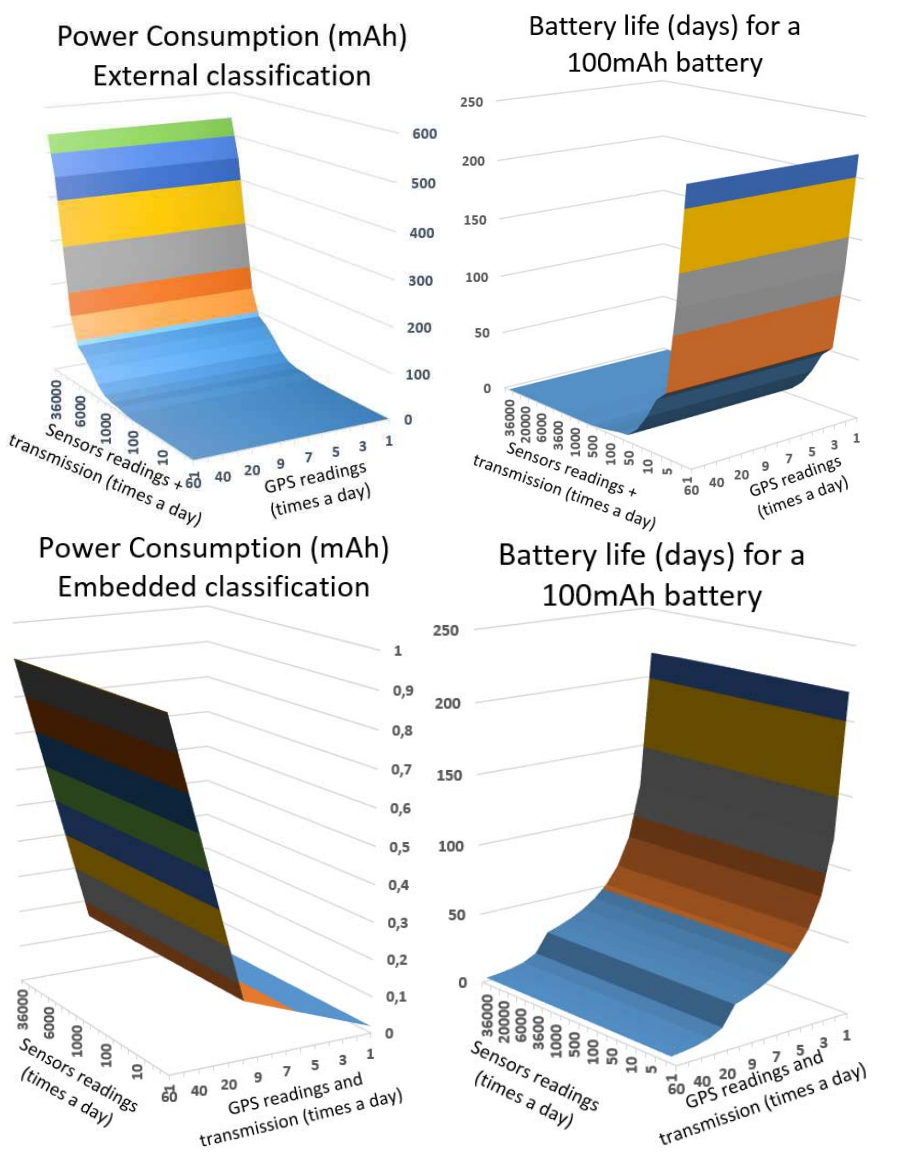

Fig. 3. Power consumption (left) and battery life (right) for External classification (top) and Neural Network (embedded) classification (bottom). GPS values: 1-60, Sensor values: 1-36000.

sensors readings start; after that, the neural network classifies the behavior (depending on the actual values and the previous ones) and stores it. Sensors, GPS and transmission can be configured to be read with different periodicities.

Two testing scenarios have been evaluated:

- External classification: each time the sensors are read, the system transmits the entire information using the XBee module; thereby, the periodicity of the transmission is the same as the sensor reads. On the other hand, the GPS read periodicity can be configured separately. Power consumption results are shown in Fig. 3 top, with different periodicity values of sensors+transmission and GPS.

- Embedded classification: the transmission periodicity is configured separately from the sensors reads; the GPS has the same periodicity as the transmissions. Power consumption results can be seen in Fig. 3 bottom, with different periodicity values of transmission+GPS and sensors.

Power consumption and battery life are almost the same in the best cases. However, to obtain a valid behavior classification, a sensor reading periodicity of $1 \mathrm{read} / \mathrm{second}$ (or 0.5 readings/second) is needed, i.e. between 36,000 and 86,000 reads a day. For high periodicity values, the neural network solution obtains much better power consumption results (see Fig. 4 for battery life comparison).

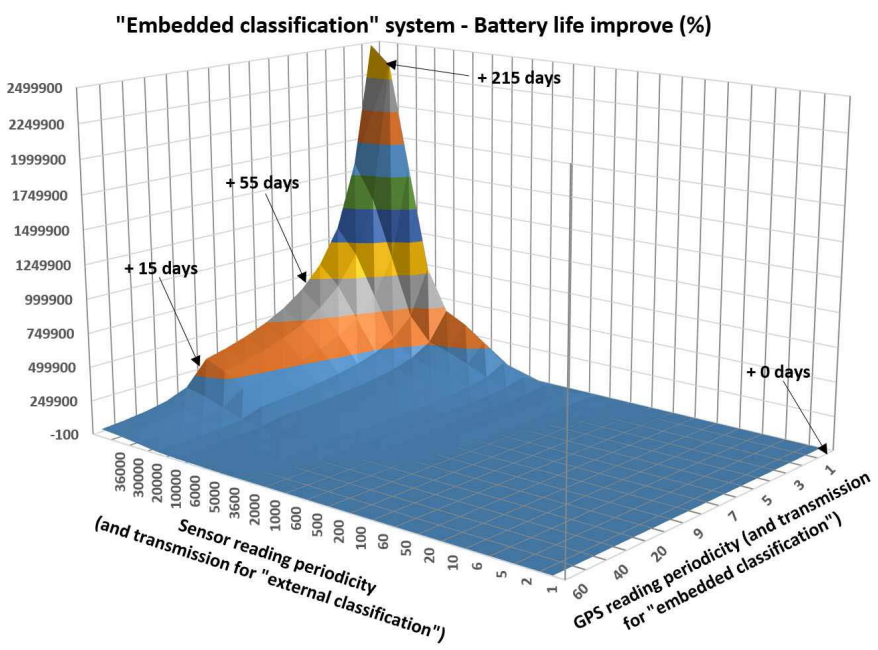

Fig. 4. Battery life increase (\%) between neural network classification method and external classification method. $1 \mathrm{read} / \mathrm{second}$ for sensors has more than $100000 \%$ increase in battery life (since transmission takes $85 \%$ of power consumption).

\section{CONCLUSION}

This work presents a WSN, implemented in a distributed system with a sensory fusion integration in order to study and classify animal behaviors in real time, which is a novelty for animal tracking networks. We have presented a network topology that allows this kind of processing of the information obtained from the sensory fusion, and the communication between every element on it. A power consumption study is presented to compare embedded and external classifications. Best battery life is obtained when data is sent after a classification step using a neural network embedded in the collar. Testing results for signal coverage are presented.

\section{REFERENCES}

[1] M. Dominguez-Morales et al., "Technical viability study for behavioral monitoring of wildlife animals in Doñana," in Proc. ICETE, 2011, pp. 1-4.

[2] E. Painkras et al., "SpiNNaker: A multi-core system-on-chip for massively-parallel neural net simulation," in Proc. IEEE Custom Int. Circuits Conf., Sep. 2012, pp. 1-4.

[3] R. T. Morales et al., "System based on inertial sensors for behavioral monitoring of wildlife," in Proc. Comput. Inf. Telecommun. Syst. (CITS), Jul. 2015, pp. 1-4.

[4] Doñana National Park, accessed on 2016. [Online]. Available: http://whc.unesco.org/en/list/685

[5] 15.4: Low-Rate Wireless Personal Area Networks (LR-WPANs), IEEE Standard 802.15.4, 2011.

[6] STM32F411RE. [Online]. Available: http://www.st.com/resource/en/ datasheet/stm32f411vc, 2016.

[7] T. S. Rappaport, Wireless Communications: Principles and Practice. Englewood Cliffs, NJ, USA: Prentice-Hall, 1996.

[8] TelosB. [Online]. Available: http://www.advanticsys.com/wiki/ index.php?title=CM5000-SMA, 2016

[9] I. Guvenc et al., "Enhancements to RSS based indoor tracking systems using Kalman filters," in Proc. Int. Signal Conf., 2003, pp. $91-102$.

[10] R. E. Kalman, "A new approach to linear filtering and prediction problems," J. Basic Eng., vol. 82, no. 1, pp. 35-45, Nov. 1960.

[11] S. Madgwick, "An efficient orientation filter for inertial and inertial/magnetic sensor arrays," Rep. x-io Univ. Bristol, U.K., Tech. Rep., 2010 .

[12] E. Cerezuela-Escudero et al., "Performance evaluation of neural networks for animal behaviors classification: Horse gaits case study," in Proc. 13th Int. Conf. Distrib. Comput. Artif. Intell. (DCAI), 2016, pp. 377-385. 